PROCEEDINGS OF THE

AMERICAN MATHEMATICAL SOCIETY

Volume 138, Number 10, October 2010, Pages 3717-3722

S 0002-9939(10)10360-8

Article electronically published on April 13, 2010

\title{
FIXED POINTS AND PERIODIC POINTS OF ORIENTATION-REVERSING PLANAR HOMEOMORPHISMS
}

\author{
J. P. BOROŃSKI \\ (Communicated by Bryna Kra) \\ Dedicated to the memory of Professor Andrzej Lasota (1932-2006)
}

\begin{abstract}
Two results concerning orientation-reversing homeomorphisms of the plane are proved. Let $h: \mathbb{R}^{2} \rightarrow \mathbb{R}^{2}$ be an orientation-reversing planar homeomorphism with a continuum $X$ invariant (i.e. $h(X)=X$ ). First, suppose there are at least $n$ bounded components of $\mathbb{R}^{2} \backslash X$ that are invariant under $h$. Then there are at least $n+1$ components of the fixed point set of $h$ in $X$. This provides an affirmative answer to a question posed by $\mathrm{K}$. Kuperberg. Second, suppose there is a $k$-periodic orbit in $X$ with $k>2$. Then there is a 2-periodic orbit in $X$, or there is a 2-periodic component of $\mathbb{R}^{2} \backslash X$. The second result is based on a recent result of M. Bonino concerning linked periodic orbits of orientation-reversing homeomorphisms of the 2-sphere $\mathbb{S}^{2}$. These results generalize to orientation-reversing homeomorphisms of $\mathbb{S}^{2}$.
\end{abstract}

\section{INTRODUCTION}

Let $h: \mathbb{R}^{2} \rightarrow \mathbb{R}^{2}$ be an orientation-reversing homeomorphism of the plane onto itself with a continuum $X$ invariant (i.e. $h(X)=X$ ). Suppose there are at least $n$ bounded components of $\mathbb{R}^{2} \backslash X$ that are invariant under $h$. In 1989 Krystyna Kuperberg [10] asked whether $h$ must always have $n+1$ fixed points in $X$. Earlier, in 1978, Harold Bell [1] showed that this is true for $n=0$. Kuperberg 9] proved this result for $n=1$. Subsequently, she also showed 10 that $h$ must have at least $k+2$ fixed points in $X$, whenever $n \geq 2^{k}$. Drawing on ideas from [9] and [10] we will present an affirmative answer to the above question. More precisely, we will prove the following stronger result.

Theorem 1.1. Let $h: \mathbb{R}^{2} \rightarrow \mathbb{R}^{2}$ be an orientation-reversing homeomorphism of the plane onto itself with a continuum $X$ invariant, and suppose there are at least $n$ bounded components of $\mathbb{R}^{2} \backslash X$ that are invariant under $h$. Then Fix $(X, h)$, the set of fixed points of $h$ in $X$, has at least $n+1$ components.

In the present paper we also discuss another problem concerning periodic points of orientation-reversing homeomorphisms. Recently, Marc Bonino [2] showed that if $h: \mathbb{S}^{2} \rightarrow \mathbb{S}^{2}$ is an orientation-reversing homeomorphism of $\mathbb{S}^{2}$ onto itself with an orbit $\mathcal{O}$ of period $k>2$, then $h$ must also have an orbit $\mathcal{O}^{\prime}$ of period 2. Using

Received by the editors August 8, 2009 and, in revised form, December 31, 2009.

2010 Mathematics Subject Classification. Primary 55M20; Secondary 54F15, 54H25, 58C30.

Key words and phrases. Fixed point, periodic point, planar homeomorphism, continuum.

The author was supported in part by NSF Grant \#DMS0634724.

(C)2010 American Mathematical Society 
Nielsen's theory he strengthened his result in [3], showing that if $h$ has a $k$-periodic orbit $\mathcal{O}$ with $k>2$, then there is a 2-periodic orbit $\mathcal{O}^{\prime}$ such that $\mathcal{O}$ and $\mathcal{O}^{\prime}$ are linked. Two orbits $\mathcal{O}$ and $\mathcal{O}^{\prime}$ are linked in the sense of Bonino if one cannot find a Jordan curve $C \subseteq \mathbb{S}^{2}$ separating $\mathcal{O}$ and $\mathcal{O}^{\prime}$ which is freely isotopic to $h(C)$ in $\mathbb{S}^{2} \backslash\left(\mathcal{O} \cup \mathcal{O}^{\prime}\right) . C$ and $h(C)$ are freely isotopic in $\mathbb{S}^{2} \backslash\left(\mathcal{O} \cup \mathcal{O}^{\prime}\right)$ if there is an isotopy $\left\{i_{t}: \mathbb{S}^{1} \rightarrow \mathbb{S}^{2} \backslash\left(\mathcal{O} \cup \mathcal{O}^{\prime}\right): 0 \leq t \leq 1\right\}$ from $i_{0}\left(\mathbb{S}^{1}\right)=C$ to $i_{1}\left(\mathbb{S}^{1}\right)=h(C)$; i.e. $i_{t}\left(\mathbb{S}^{1}\right)$ is a Jordan curve for any $t\left(\mathbb{S}^{1}\right.$ denotes the unit circle). Exploiting heavily results from the second paper we will show the following.

Theorem 1.2. Let $h: \mathbb{R}^{2} \rightarrow \mathbb{R}^{2}$ be an orientation-reversing homeomorphism with a continuum $X$ invariant (i.e. $h(X)=X$ ). Suppose $h$ has a $k$-periodic orbit in $X$ with $k>2$.

(i) If $X$ does not separate the plane, then $h$ has a 2-periodic orbit in $X$.

(ii) If $X$ separates the plane, then $h$ has a 2-periodic orbit in $X$, or there is a 2-periodic component $U$ of $\mathbb{R}^{2} \backslash X$.

The above result seems to be related to a special case of the Sarkovskii Theorem 11, which asserts that a self-map of the arc that has a point of period $k>2$ must also have a point of period 2 .

\section{Preliminaries}

Given a set $D$, by Int $D$ and $\partial D$ we will denote respectively the interior and the boundary of $D$. Throughout this paper $h$ is an orientation-reversing homeomorphism of the plane $\mathbb{R}^{2}$ onto itself and $X$ is a continuum (i.e. connected and compact subset of the plane) invariant under $h$; that is, $h(X)=X$. Denote by $F i x(X, h)$ the set of fixed points of $h$ in $X$; i.e. Fix $(X, h)=\{x \in X: h(x)=x\}$. Components of $\mathbb{R}^{2} \backslash X$ are called complementary domains of $X$. A point $x$ (a complementary domain $U$ of $X)$ is $k$-periodic if $h^{k}(x)=x$ but $h^{p}(x) \neq x\left(h^{k}(U)=U\right.$ but $\left.h^{p}(U) \neq U\right)$ for any positive integer $p<k$. $\mathcal{O}$ is a $k$-periodic orbit if $\mathcal{O}=\left\{x, h^{1}(x), \ldots, h^{k-1}(x)\right\}$ for a $k$-periodic point $x$. Let us recall the methods of [9] and [10] that we will rely on in order to prove Theorem 1.1. Let $U$ be a bounded complementary domain of $\mathbb{R}^{2} \backslash X$ that is invariant under $h$. With modification of $h$ outside of $X$ one can ensure that there is an annulus $\mathbb{A}$ invariant under $h$ such that $X \subseteq \mathbb{A}$. $\mathbb{A}$ is topologically a geometric annulus $\left\{(r, \theta) \in \mathbb{R}^{2}: 1 \leq r \leq 2,0 \leq \theta<2 \pi\right\}$, given in polar coordinates, with two boundary components $\mathbb{A}^{+}=\left\{(r, \theta) \in \mathbb{R}^{2}: r=2,0 \leq \theta<2 \pi\right\}$ and $\mathbb{A}^{-}=\left\{(r, \theta) \in \mathbb{R}^{2}: r=1,0 \leq \theta<2 \pi\right\}$. The continuum $X$ is essentially inscribed into $\mathbb{A}$; i.e. $\mathbb{A}^{-} \subseteq U$. Now, one can consider the universal covering space of $\mathbb{A}$ given by $\tilde{\mathbb{A}}=\left\{(x, y) \in \mathbb{R}^{2}: 1 \leq y \leq 2\right\}$, with the covering map $\tau: \widetilde{\mathbb{A}} \rightarrow \mathbb{A}$ determined by $\tau(x, y)=(y, 2 \pi x(\bmod 2 \pi))$. Let $\tilde{h}: \tilde{\mathbb{A}} \rightarrow \tilde{\mathbb{A}}$ be a lift homeomorphism of $h \mid \mathbb{A}$ (i.e. $\tau \circ h=\tilde{h} \circ \tau)$. Note that for any $p=(r, \theta)$ in $\mathbb{A}$ its fiber is the set $\tau^{-1}(p)=\left\{\left(\frac{\theta}{2 \pi}+n, r\right): n \in \mathbb{Z}\right\}$, and $p$ is a fixed point of $h$ iff $\tau^{-1}(p)$ is invariant under $\tilde{h}$. The main ingredients from [9] and [10] that we will need are the following facts.

(1) Given a fixed point $p=(r, \theta) \in \mathbb{A}$ and a lift $\tilde{h}$ of $h$ there is an integer $m[\tilde{h}, p]$ such that $\tilde{h}\left(\frac{\theta}{2 \pi}+n, r\right)=\left(\frac{\theta}{2 \pi}-n+m[\tilde{h}, p], r\right)$ for every $\left(\frac{\theta}{2 \pi}+n, r\right) \in \tau^{-1}(p)$.

(2) $\tilde{h}$ has a fixed point in $\tau^{-1}(p)$ iff $m[\tilde{h}, p]$ is even.

(3) If $m[\tilde{h}, p]$ is even, then $\tilde{h}(x+1, y)$ is a lift homeomorphism of $h$ that does not have a fixed point in $\tau^{-1}(p)$. 
(4) $\tilde{\mathbb{A}}$ can be compactified by two points, say $b_{1}, b_{2}$, so that $\tilde{X}=\tau^{-1}(X) \cup$ $\left\{b_{1}, b_{2}\right\}$ is a continuum invariant under $\tilde{h}$, and the latter can be extended to an orientation-reversing homeomorphism of the entire plane onto itself.

Let $\tilde{h_{1}}: \tilde{\mathbb{A}} \rightarrow \tilde{\mathbb{A}}$ be a lift of $h$ and $\tilde{h}_{2}(x, y)=\tilde{h}_{1}(x+1, y)$ be another lift, fixed once and for all. For simplicity we will use the same symbols $\tilde{h}_{1}, \tilde{h}_{2}$ to denote the extensions of these two lifts to the entire plane.

Proposition 2.1. If $Y$ is a subcontinuum of the set of fixed points of $h$, then $Y$ does not separate the plane.

Proof. If $F$ is the fixed point set of a homeomorphism $f$ of a connected topological manifold $M$, then either each component of $M \backslash F$ is invariant under $f$ or there are exactly two components of $M \backslash F$ and $f$ interchanges them [6]. Since in the case of planar homeomorphisms the unbounded complementary domain of $F$ is always invariant under $h$, therefore the above implies that all components of $\mathbb{R}^{2} \backslash F$ must be invariant under $h$. Consequently if $Y$ were a continuum of fixed points of $h$ separating the plane, then $Y$ could be essentially inscribed into the annulus $\mathbb{A}$ with $\mathbb{A}^{-}$and $\mathbb{A}^{+}$invariant under $h$, and $h$ would induce the identity on the homology group $\mathrm{H}_{1}(\mathbb{A}, \mathbb{Z})$. Therefore any lift $\tilde{h}$ of $h$ to the universal cover $\tilde{\mathbb{A}}$ would preserve the orientation on the two boundary components of $\tilde{\mathbb{A}}$, at the same time keeping them invariant. Consequently $\tilde{h}$ would be orientation-preserving on $\tilde{\mathbb{A}}$, contradicting the fact that any lift of $h$ to $\tilde{\mathbb{A}}$ must be orientation-reversing.

Lemma 2.2. Suppose $p$ is a fixed point of $h$ and let $Y$ be the component of $p$ in $\operatorname{Fix}(X, h)$. Then

for every $q \in Y$.

$$
m\left[\tilde{h}_{1}, p\right]=m\left[\tilde{h}_{1}, q\right](\bmod 2)
$$

Proof. First, $Y$ does not separate the plane. Suppose $m\left[\tilde{h}_{1}, p\right]$ is even. Let $\alpha$ be the fixed point of $\tilde{h}_{1}$ in $\tau^{-1}(p)$ and let $K$ be the component of $\tau^{-1}(Y)$ containing $\alpha$. To the contrary, suppose the above claim is false and let $q \in Y$ be such that $m\left[\tilde{h}_{1}, q\right]$ is odd. For every $\beta \in \tau^{-1}(q)$ we have $\beta \neq \tilde{h}_{1}(\beta)$. Let $\gamma$ be in $K \cap \tau^{-1}(q)$. Then $\tilde{h}_{1}(\gamma) \neq \gamma$ and $\tilde{h}_{1}(\gamma) \in \tilde{h}_{1}(K)$. Since $\tilde{h}_{1}(K)$ is also a component of $\tau^{-1}(Y)$ and $\alpha \in \tilde{h}_{1}(K)$, then $K=\tilde{h}_{1}(K)$. Consequently, $K$ contains two elements from the same fiber $\tau^{-1}(q) \in \tau^{-1}(Y)$. But this contradicts the following observation indicated in [5], which in turn will complete the proof.

Since $Y \subseteq \mathbb{A}$ does not separate the plane, one can choose a disk $D \subseteq \mathbb{A}$ around $Y$; i.e., $Y \subseteq \operatorname{Int} D$, and Int $D$ being simply connected lifts to disjoint homeomorphic copies of Int $D$ in $\tilde{\mathbb{A}}$. Consequently $Y$ lifts to disjoint homeomorphic copies in $\tilde{\mathbb{A}}$. Since $K$ is one of them, it cannot contain two points from the same fiber $\tau^{-1}(q)$.

As a consequence of the above, for a given component $Y$ of Fix $(X, h)$ one can choose any $p \in Y$ and say that $m\left[\tilde{h}_{1}, Y\right]$ is even (or odd) if $m\left[\tilde{h}_{1}, p\right]$ is of the same parity.

\section{Proof of Theorem 1.1}

Proof of Theorem 1.1. We will prove this theorem by induction. First, observe that the case when $n=0$ is the theorem of Bell [1]. Indeed, if $X$ is a nonseparating plane continuum, then by Bell's theorem $h$ must have a fixed point in $X$, and therefore there is at least one component of $\operatorname{Fix}(X, h)$. 
For the sake of induction suppose the theorem is true for $n=k-1$. Now we will show that the theorem holds true for $n=k$.

Assume $U_{1}, \ldots, U_{k}$ are bounded complementary domains of $X$ invariant under $h$, and that $\mathbb{A}^{-}$is inscribed into $U_{k}$. We may assume that there is a fixed point $u_{i}$ of $h$ in each $U_{i}$. Without loss of generality assume that $u_{1}, \ldots, u_{p}$ are all fixed points of $h$ such that there is a fixed point of $\tilde{h}_{1}$ in the fiber $\tau^{-1}\left(u_{i}\right)$, for all $i=1, \ldots, p$. In other words, each set from $U_{1}, \ldots, U_{p}$ contains in its lift $\tau^{-1}\left(U_{i}\right)$ a bounded complementary domain of $\tilde{X}$ that is invariant under $\tilde{h}_{1}$. Equivalently, $m\left[\tilde{h}_{1}, u_{i}\right]$ is even for $i=1, \ldots, p$ and $m\left[\tilde{h}_{1}, u_{i}\right]$ is odd for $i=p+1, \ldots, k-1$.

Let $q=k-1-p$. Note that $p, q$ are nonnegative integers (possibly with $p$ or $q$ equal to 0$)$. Since $\tilde{X}$ is a continuum with $p$ bounded complementary domains invariant under $\tilde{h}_{1}$ and $p \leq k-1$, by the induction hypothesis there are $p+1$ components of $\operatorname{Fix}\left(\tilde{h}_{1}, \tilde{X}\right)$. Let $A_{1}, \ldots, A_{p+1}$ be those components.

For every $i=1, \ldots, p+1$ there is a component $X_{i}$ of Fix $(h, X)$ such that $X_{i}=\tau\left(A_{i}\right)$. Note that $\tau\left(A_{i}\right)$ and $\tau\left(A_{t}\right)$ are disjoint for $i \neq t$ since any fiber of a fixed point of $h$ contains no more that one fixed point of $\tilde{h}_{1}$. Therefore $\left\{X_{i}: i=\right.$ $1, \ldots, p+1\}$ consists of $p+1$ distinct components of $F i x(X, h)$.

Now, $\tau^{-1}\left(X_{i}\right)$ is invariant under $\tilde{h}_{1}$, and $m\left[\tilde{h}_{1}, X_{i}\right]$ is even for every $i=1, \ldots$, $p+1$. $\tau^{-1}\left(X_{i}\right)$ is also invariant under $\tilde{h}_{2}$ but contains no fixed point of $\tilde{h}_{2}$, since $m\left[\tilde{h}_{2}, X_{i}\right]$ is odd for every $i=1, \ldots, p+1$. For $i=1, \ldots, p$, no $\tau^{-1}\left(u_{i}\right)$ contains a fixed point of $\tilde{h}_{2}$, since $m\left[\tilde{h}_{2}, u_{i}\right]$ is odd. For $i=p+1, \ldots, k-1$, every $\tau^{-1}\left(u_{i}\right)$ contains a fixed point of $\tilde{h}_{2}$, since $m\left[\tilde{h}_{2}, u_{i}\right]$ is even. Therefore, there are $q=$ $(k-1)-p$ bounded complementary domains of $\tilde{X}$ that are invariant under $\tilde{h}_{2}$. Again, by the induction hypothesis, there must be $q+1$ components of $F i x\left(\tilde{h}_{2}, \tilde{X}\right)$. Denote them by $C_{1}, \ldots, C_{q}$. For every $j=1, \ldots, q+1, \tau\left(C_{j}\right)$ is a component of $F i x(h, X)$. Note that $\tau\left(C_{j}\right)$ and $\tau\left(C_{t}\right)$ are disjoint for $j \neq t$ since any fiber of a fixed point of $h$ contains no more that one fixed point of $\tilde{h}_{2}$. Therefore $\left\{\tau\left(C_{j}\right): j=1, \ldots, q+1\right\}$ consists of $q+1$ distinct components of $F i x(X, h)$. Since each $\tau^{-1}\left(X_{i}\right)$ contains no fixed point of $\tilde{h}_{2}$, no $\tau\left(C_{j}\right)$ can coincide with any $X_{i}$. Therefore there are $p+1+q+1=k+1$ components of Fix $(h, X)$. This completes the proof.

Note that Theorem 1.1 generalizes to orientation-reversing homeomorphisms of $\mathbb{S}^{2}$. More precisely, we get the following as a corollary.

Theorem 3.1. Let $g: \mathbb{S}^{2} \rightarrow \mathbb{S}^{2}$ be an orientation-reversing homeomorphism of $\mathbb{S}^{2}$ onto itself with a continuum $X$ invariant, and suppose there are at least $n$ components of $\mathbb{S}^{2} \backslash X$ that are invariant under $g$. Then Fix $(X, g)$ has at least $n$ components.

Proof. First suppose that $\mathbb{S}^{2} \backslash X$ has exactly one component $U$ invariant under $g$. We can assume that there is a fixed point $u$ of $g$ in $U$. Notice that $\mathbb{S}^{2} \backslash\{u\}$ is topologically the plane, and $G=g \mid\left(\mathbb{S}^{2} \backslash\{u\}\right)$, obtained by the restriction of $g$ to $\mathbb{S}^{2} \backslash\{u\}$, is an orientation-reversing homeomorphism of the plane onto itself with the continuum $X$ invariant. Now, since $X$ has no bounded complementary domains invariant under $G$, by a theorem of Bell there is at least one component of $F i x(X, G)=F i x(X, g)$. Bell's theorem applies to nonseparating plane continua, but in the above case if $X$ separates the plane and none of the bounded complementary domains is invariant under $G$, then these domains can be added to $X$ to form a nonseparating plane continuum $Y$ with $\operatorname{Fix}(X, G)=\operatorname{Fix}(Y, G)$. 
Second, suppose that $\mathbb{S}^{2} \backslash X$ has at least two components $U_{1}$ and $U_{2}$ invariant under $g$. Then there is an annulus $\mathbb{A}$ such that $X \subseteq \mathbb{A}, \mathbb{A}^{-} \subseteq U_{1}$ and $\mathbb{A}^{+} \subseteq U_{2}$. Since $U_{1}$ and $U_{2}$ are invariant under $g$, then $h$ does not interchange $\mathbb{A}^{-}$and $\mathbb{A}^{+}$, and one can repeat the proof of Theorem 1.1

\section{Proof of Theorem 1.2}

Theorem 1.2 seems to fit well in the following context. The Cartwright-Littlewood-Bell theorem (see 7] and [1]) states that any planar homeomorphism fixes a point in an invariant nonseparating continuum. Morton Brown [5] and O.H. Hamilton 8] exhibited that, in the case of orientation-preserving homeomorphisms, this theorem can be deduced directly from a theorem of Brouwer [4. Brouwer showed that any orientation-preserving homeomorphism with at least one bounded orbit must have a fixed point. Briefly, the idea behind these short proofs of the fixed point theorem was to separate the invariant continuum from the fixed-point set $F$, and then for an open invariant component $U$ in $\mathbb{R}^{2} \backslash F$ containing $X$ argue that $U$ contains no fixed point, thus contradicting the theorem of Brouwer. The inspiration for the proof of Theorem 1.2 comes from these very papers, but since the set of 2-periodic points does not need to be closed (in contrast with the fixed-point set), one cannot just replace the theorem of Brouwer with a theorem of Bonino from [2] and use the same arguments. Instead, we will use Bonino's result from [3] and show that no 2-periodic orbit in an invariant component of $\mathbb{R}^{2} \backslash X$ can be linked to a $k$-periodic $(k>2)$ orbit in $X$.

Proof of Theorem 1.2. Compactify $\mathbb{R}^{2}$ by a point $\infty$ to obtain $\mathbb{S}^{2}=\mathbb{R}^{2} \cup\{\infty\}$ and extend the given homeomorphism $h: \mathbb{R}^{2} \rightarrow \mathbb{R}^{2}$ to a homeomorphism $\tilde{h}: \mathbb{S}^{2} \rightarrow \mathbb{S}^{2}$ by setting $\tilde{h} \mid \mathbb{R}^{2}=h$ and $\tilde{h}(\infty)=\infty$. $h$ and $\tilde{h}$ have exactly the same $k$-periodic points for any $k>1$.

By Bonino's result there is an orbit $\mathcal{O}^{\prime} \subseteq \mathbb{S}^{2}$ of $\tilde{h}$ of period exactly 2. We will show that any such 2-periodic orbit that lies in an invariant complementary domain of $X$ is not linked to $\mathcal{O}$.

Suppose $\mathcal{O}^{\prime} \cap X=\emptyset$ and $\mathcal{O}^{\prime} \subseteq U$ for a complementary domain $U$ of $X$ invariant under $h$. Since $\mathcal{O}^{\prime}$ and $X$ are closed, there is a Jordan curve $S \subseteq \mathbb{S}^{2}$ separating $\mathcal{O}^{\prime}$ from $X$. Let $D$ be one of the two disks in $\mathbb{S}^{2}$ bounded by $S$, such that $X \subseteq \operatorname{Int} D$. Then $D \cap \mathcal{O}^{\prime}=\emptyset$. Since $X$ is invariant under $\tilde{h}$, by continuity of $\tilde{h}$, there is a disk $C$ such that $C \subseteq \operatorname{Int} D$ and $\tilde{h}(C) \subseteq$ Int $D$. Since both $C$ and $\tilde{h}(C)$ contain $X$ in its interior, there is a disk $B \subseteq C \cap \tilde{h}(C)$ that contains $X$ in its interior. Therefore $C$ and $\tilde{h}(C)$ are freely isotopic in the annulus $D \backslash \operatorname{Int} B$, thus freely isotopic in $\mathbb{S}^{2} \backslash\left(\mathcal{O} \cup \mathcal{O}^{\prime}\right)$. This shows that if $\mathcal{O}^{\prime} \subseteq \mathbb{S}^{2} \backslash X$ is a 2-periodic orbit, then $\mathcal{O}^{\prime}$ and $\mathcal{O}$ are not linked. Therefore the 2-periodic orbit $\mathcal{O}^{\prime}$ linked to $\mathcal{O}$, guaranteed by a theorem of Bonino in [3, must be in $X$ or in a 2-periodic component of $\mathbb{S}^{2} \backslash X$.

Corollary 4.1. Suppose there is a k-periodic component of $\mathbb{R}^{2} \backslash X$, for $k>2$. Then either there is a 2-periodic orbit in $X$ or there is a 2-periodic component of $\mathbb{R}^{2} \backslash X$.

Proof. Let $W$ be a $k$-periodic complementary domain of $X(k>2)$. Without loss of generality one may assume that there is a $k$-periodic point $w$ in $W$ ( $w$ is a fixed point of $\left.h^{k}\right)$. Consider $Y=X \cup W \cup h(W) \cup \ldots \cup h^{k-1}(W)$. Clearly $Y$ is a continuum invariant under $h$. Now apply Theorem 1.2. 
Remark. It is clear from the proof of Theorem 1.2 that this theorem holds also for any orientation-reversing homeomorphism of $\mathbb{S}^{2}$. On the other hand, it is not apparent to the present author if one can improve Theorem 1.2 and get rid of the 2-periodic component of $\mathbb{R}^{2} \backslash X$ to guarantee that, under the assumptions, there will be a 2-periodic point in $X$. Nonetheless, the following example shows that one cannot do it for $\mathbb{S}^{2}$.

Example. Let $\mathbb{S}^{2}$ be given in spherical coordinates by $\mathbb{S}^{2}=\{(r, \theta, \phi): r=1,0 \leq$ $\theta<2 \pi, 0 \leq \phi \leq \pi\}$. Consider the Jordan curve $S \subseteq \mathbb{S}^{2}$ determined by $S=$ $\left\{(r, \theta, \phi): r=1, \phi=\frac{\pi}{2}\right\}$. Let $U^{+}, U^{-}$be the two disks in $\mathbb{S}^{2} \backslash S$ bounded by $S$. Fix $k>2$ and consider the orientation-reversing homeomorphism $g: \mathbb{S}^{2} \rightarrow \mathbb{S}^{2}$ determined by

$$
g(r, \theta, \phi)=\left(r, \theta+\frac{2 \pi}{k}, \pi-\phi\right) .
$$

$g$ interchanges $U^{+}$and $U^{-}$, reflecting $\mathbb{S}^{2}$ about $S$ and then rotating $\mathbb{S}^{2}$ by $\frac{2 \pi}{k}$. Notice that $g^{2}(r, \theta, \phi)=\left(r, \theta+\frac{4 \pi}{k}, \phi\right)$ and $g^{k}(r, \theta, \phi)=(r, \theta, \phi)=i d_{\mathbb{S}^{2}}(r, \theta, \phi)$. Clearly, $g$ is an orientation-reversing homeomorphism of $\mathbb{S}^{2}$ with the continuum $S$ invariant, and any point in $S$ is of period exactly $k$, but the only points of period 2 are the two poles, which are not in $S$.

\section{ACKNOWLEDGMENT}

The author is indebted to his Ph.D. advisor, Professor Krystyna Kuperberg, for bringing the question from [10] to his attention, for a patient reading of several preliminary notes on a solution of the problem, and for many valuable comments.

\section{REFERENCES}

1. H. BeLL, A fixed point theorem for plane homeomorphisms. Fund. Math. 100 (1978), 119-128. MR0500879 (58:18386)

2. M. Bonino, A Brouwer-like theorem for orientation reversing homeomorphisms of the sphere. Fund. Math. 182 (2004), no. 1, 1-40. MR2100713 (2005m:37096)

3. M. Bonino, Nielsen theory and linked periodic orbits of homeomorphisms of $\mathbb{S}^{2}$. Math. Proc. Cambridge Philos. Soc. 140 (2006), no. 3, 425-430. MR2225641 (2007k:37054)

4. L. E. Brouwer, Beweis des Ebenen Translationssatzes, Math. Ann. 72 (1912), 36-54.

5. M. Brown, A short short proof of the Cartwright-Littlewood theorem. Proc. Amer. Math. Soc., 65, no. 2 (1977), 372. MR0461491 (57:1476)

6. M. Brown, J. M. Kister, Invariance of complementary domains of a fixed point set. Proc. Amer. Math. Soc. 91 (1984), no. 3, 503-504. MR744656 (86c:57014)

7. M. L. Cartwright, J. E. Littlewood, Some fixed point theorems. With appendix by H. D. Ursell, Ann. of Math. (2) 54 (1951), 1-37. MR0042690(13:148f)

8. O. H. Hamilton, A short proof of the Cartwright-Littlewood fixed point theorem. Canadian J. Math. 6 (1954), 522-524. MR0064394 (16:276a)

9. K. KuperberG, Fixed points of orientation reversing homeomorphisms of the plane. Proc. Amer. Math. Soc. 112 (1991), no. 1, 223-229. MR.1064906 (91h:54049)

10. K. Kuperberg, A lower bound for the number of fixed points of orientation reversing homeomorphisms. The geometry of Hamiltonian systems (Berkeley, CA, 1989), 367-371, Math. Sci. Res. Inst. Publ., 22, Springer, New York, 1991. MR.1123283 (92j:54044)

11. O. M. SARKovSKII, Co-existence of cycles of a continuous mapping of the line into itself. Ukrain. Mat. Z. 16 (1964), 61-71. MR0159905 (28:3121)

Department of Mathematics and Statistics, Auburn University, Auburn, Alabama 36849

E-mail address: boronjp@auburn.edu 